\title{
Conferencia inaugural "Doctora María Luisa Chavoya Peña"
}

DOI: $\underline{\text { https://doi.org/10.32870/dse.v0i12.266 }}$

\section{Mercedes Palencia Villa*}

Como todos los inicios del semestre, se realiza una conferencia inaugural dirigida a los estudiantes y profesores de la academia de sociología y educación, sobre algún tema relevante que resulte de su interés; sin embargo, a diferencia de las anteriores ocasiones, hoy resulta de especial importancia este ritual, ya que a partir de este momento la conferencia se denominará "María Luisa Chavoya Peña”, y este acontecimiento quedará en la memoria de los estudiantes que se inscriben en esta especialidad. Cabe señalar que, aunque no hay autoridades universitarias, ni placas conmemorativas que denominen oficialmente esta designación, esto se debe a que se trata de una propuesta de sus colegas más allegados, quienes la conocieron y pudieron constatar su trayectoria académica, además de su trabajo incansable. Debido a lo anterior, sería interesante preguntarnos sobre el significado que tiene en la historia de las instituciones, asociar una acción académica con el nombre de una persona. Habría que preguntarnos: ¿por qué resulta importante dejar en la memoria colectiva de una institución educativa el nombre de una persona?

Debido a que las instituciones no son entelequias que puedan estudiarse de manera directa, pues existen zonas oscuras que pertenecen al mundo de lo simbólico, la legitimación que puede lograr una institución es a través de la transmisión a las nuevas generaciones de discursos y acciones simbólicas. En este sentido, analizar la trayectoria de personas insertadas en la institución nos ayuda a conocer que nuestro quehacer cotidiano contribuye a la configuración institucional. Sin embargo, resulta necesario que permanezca el mundo institucional como una realidad objetiva, y esto es posible a partir de la historia institucional, ya que forma parte de la tradición de las instituciones existentes y le otorga un carácter de objetividad. Así pues, institucionalizar esta conferencia como "María Luisa Chavoya Peña" implica legitimar el trabajo académico que realizó nuestra compañera durante más de treinta años, con la finalidad de que estas legitimaciones sean aprendidas por las nuevas generaciones durante el mismo proceso que las socializan dentro del orden institucional.

* Doctora en educación. Profesora investigadora del Departamento de Estudios en Educación, Universidad de Guadalajara. Correo electrónico: barcelona.mercedes@gmail.com 
La función de la legitimación 1 , según Berger y Luckman (1994: 120), "consiste en lograr que las objetivaciones de primer orden ya institucionalizadas, lleguen a ser objetivamente disponibles y subjetivamente plausibles". De ahí que el problema de la legitimación surge cuando las objetivaciones del orden institucional (ahora histórico), deben transmitirse a una nueva generación ${ }^{2}$.

Lo que puede unir a María Luisa con las generaciones actuales es la sociología y su legado académico. Por lo tanto, en un primer momento analizaré las características de su profesión como socióloga, esto con la finalidad de retomar algunos de sus trabajos académicos que la condujeron a conformar la academia de sociología y educación, temática que nos convoca en la presente conferencia.

Lo anterior resulta importante dentro de los fenómenos sociales, porque esta dimensión micro incorpora la interacción cotidiana para comprender las normas y los valores instituidos. Seguramente cuando los alumnos eligieron estudiar sociología, algunas personas les dijeron que se trata de una carrera poco redituable, y recuerdo que inclusive era ridiculizada y se decía: "estudias la ociología", lo cual, si lo traducimos al sentido común, significa que somos ociosos al estudiar la interacción social, como objeto de estudio. En este sentido, Bauman nos dice:

la sociología se distingue de las demás ciencias sociales, en que considera a las acciones humanas como elementos de elaboraciones más amplias, es decir, de una disposición no aleatoria de los actores, que se encuentran aprisionados en una red de dependencia mutua (siendo la dependencia un estado, en el que la probabilidad de que se realice la acción y la posibilidad de éxito, cambian en relación con lo que los actores son, hacen o pueden hacer). Los sociólogos se preguntarían qué consecuencias tendría esta interdependencia para el comportamiento real y posible de los actores humanos... Estos intereses, moldean el objeto de indagación sociológica: elaboraciones, redes de dependencia mutua, condicionamiento recíproco de la acción, expansión o limitación de la libertad de los actores: esas son las preocupaciones más importantes de la sociología (Bauman, 1990: 13).

Desde esta perspectiva, el autor afirma que todos vivimos en compañía de otras personas e interactuamos con los demás, y todo lo que la sociología abarca estuvo ya en nuestras vidas. Vivir en compañía de otras personas requiere una gran cantidad de conocimiento, y ese conocimiento se

${ }^{1}$ El término de legitimación deriva de Weber (1944: 30): "la forma de legitimidad hoy más corriente es la creencia en la legalidad: la obediencia a preceptos jurídicos positivos, estatuidos según el procedimiento usual y formalmente correctos. La contraposición entre ordenaciones pactadas y "otorgadas" es sólo relativa. Pues cuando una ordenación pactada no descansa en un acuerdo por unanimidad, no existe una legitimidad de la misma".

${ }^{2}$ Resulta importante recordar que la generación es un instrumento histórico según Ricoeur (1996), en donde todos los contemporáneos no están sometidos a las mismas influencias, por lo que resulta necesario distinguir la agrupación de localización, donde las afinidades resultan determinantes. De ahí que la noción de generación considerada por Ricoeur encuentra la conexión entre el tiempo público y el privado, fenómeno intermedio entre lo que él considera tiempo exterior del calendario y el tiempo interior de la vida psíquica. Por lo cual, lo que define una generación está determinado por un horizonte de sentido, que les permite compartir el mundo, y esto no significa que tengan la misma edad sino un modo de estar en este mundo. 
llama "sentido común". Por ello, los sociólogos están condenados a permanecer en ambos lados de la experiencia que pretenden interpretar: adentro y afuera al mismo tiempo. Por eso, trazar el límite entre el conocimiento sociológico propiamente dicho y el sentido común — que está siempre lleno de ideas sociológicas - es una cuestión importante para la identidad de la sociología, como cuerpo coherente de conocimiento. La sociología, a diferencia del sentido común, nos obliga a hacer un esfuerzo por subordinarnos a las rigurosas reglas del discurso responsable, que es un atributo de la ciencia. En el caso de María Luisa Chavoya, como señala una de sus mejores amigas:

era una socióloga de la educación en ciernes. Antes de estudiar sociología ya estudiaba los problemas educativos; su trabajo en el Departamento de Educación Pública fue como investigadora. De hecho, fue habilitada como tal, mediante un curso que tomó en la Ciudad de México. En Educación Pública, realizó diversos trabajos y diagnósticos acerca de la deserción y la reprobación en primarias de Jalisco.

Lo anterior nos indica que María Luisa estuvo adentro y afuera en el análisis de la vida social, la mayor parte de su vida. Desde muy joven, se involucró en la investigación sociológica. Por ejemplo, ella estudió previamente trabajo social, que en los años setenta no requería como antecedente la educación preparatoria, y al concluir esta carrera técnica, ingresó a la Secretaría de Educación, en donde fue capacitada en la Ciudad de México por el maestro Rojas Soriano para hacer diagnósticos. En su estancia en esta institución educativa, estudió la preparatoria nocturna, con la finalidad de ingresar a sociología, ya que cuando inició esta licenciatura tenía grandes expectativas de investigación para la primera generación. Ella se incorporó a la licenciatura en 1980 y concluyó en 1985, lo que le dio la oportunidad de ingresar muy joven aún a la universidad. Según un estudio realizado por Sonia Reynaga (1998), se consideró como la primera generación de sociología en la Universidad de Guadalajara a la que inició la carrera en 1977 y concluyó hacia finales de los ochenta. Siguiendo el estudio de dicha autora, esta generación se caracteriza por contar con mayores oportunidades para ingresar en el mercado laboral, particularmente en el área académica que se genera a partir del crecimiento de la oferta educativa que ofrece la Universidad de Guadalajara, y de la institucionalización de la actividad de investigación a mediados de los años ochenta.

El contexto que vivió María Luisa en la Facultad de Sociología es posible que influyera en sus inicios de investigación, ya que en 1985-1987 estudió la maestría en estudios regionales en el Instituto Mora de la Ciudad de México, y realizó una tesis sobre el poder sindical en Jalisco: la sección 47 del Sindicato Nacional de Trabajadores de la Educación (SNTE). El estudio pretende mostrar cómo cada grupo tiene su propia historia, que no puede equipararse ni reducirla al SNTE del ámbito nacional. Le interesó indagar sobre las relaciones clientelares como un proceso sui generis, explicable sólo a partir de la poca importancia que tuvo la burocracia educativa estatal en la consolidación del nuevo Estado nacional impulsado por el cardenismo; afirmaba que en esta sección no existe una tradición de izquierda. Esto nos conduce a concluir que su trabajo evidenció cómo el 
poder sindical se fundamenta en el control de órganos representativos y en la gestión económica y social del Estado. Este monopolio de representación ha generado estructuras verticales, que van acompañadas de prácticas de corrupción de diferente índole, que permiten la concentración de privilegios en el grupo que posee el liderazgo. Aunque se tituló en 1989, fue hasta seis años después cuando se publicó este trabajo por la Universidad de Guadalajara.

Sin embargo, en 1989 la reorientación neoliberal del Estado mexicano exigió a las universidades públicas un nuevo esquema para el financiamiento, en donde los recursos económicos vendrían desde diferentes programas académicos. Tuvo entonces como resultado un conflicto interno y una lucha política entre grupos de la universidad. Esto dio origen a la transformación de la institución en concordancia con la Reforma Educativa propuesta por el gobierno federal. Dichos cambios al interior de la universidad fueron de interés para María Luisa, y en 1994, en el Doctorado Interinstitucional con sede en Aguascalientes, elaboró otra investigación titulada La institucionalización de la investigación en la Universidad de Guadalajara.

En el año 2006 publicó un trabajo crítico en donde señala que cada vez más se implementan procedimientos rutinarios y burocráticos, que expresan una desconfianza a la profesionalización de la investigación. Estos programas de evaluación académica devalúan la competencia profesional de los investigadores, porque atenta contra la autonomía académica. Las temáticas que realizó durante su carrera fueron en torno a la Universidad de Guadalajara, y las políticas de educación superior que vivió como académica siempre fueron analizadas desde sus estudios sociológicos. Diagnósticos y análisis de la vida académica de las universidades, le permitieron analizar y participar dentro de la Universidad, en estudios que impactaron en el diseño de la Universidad de Guadalajara. Durante toda su vida participó en 25 proyectos de investigación y en total realizó 87 publicaciones, todo esto la llevó a concursar como candidata al Instituto de Evaluación de la Educación en el 2013, y a presentar su propuesta ante la Cámara de Senadores.

Desde la vida cotidiana, recuerdo que cuando ella coordinaba el doctorado y yo la maestría en educación, me enseñó los enredos administrativos que ella conocía mejor que nadie, pero al mismo tiempo, defendía una visión académica ante una práctica administrativa. Es decir, la junta académica debería responder estrictamente a la lógica académica, y no administrativa. No ejercía ninguna decisión del doctorado sin consultarlo de manera democrática con la junta académica, aunque le costara tener menos poder. Para lograrlo, era capaz de escuchar cualquier crítica de estudiantes, profesores y administrativos; además, tenía la habilidad de auto-evaluarse. Estas son prácticas que sin duda deben trascender a las nuevas generaciones de investigadores, ya que hemos vivido en las universidades un incremento de trámites administrativos, en detrimento de la discusión académica.

Debido a lo expresado anteriormente, estarán de acuerdo conmigo en que se justifica ampliamente que esta conferencia periódica lleve el nombre de María Luisa Chavoya Peña, pues la huella que dejó en la Universidad de Guadalajara trascenderá a las nuevas generaciones.

Muchas gracias. 


\section{Bibliografía}

Chavoya Peña, María Luisa (2005). Poder sindical en Jalisco: el caso de la Sección 47. Guadalajara: Universidad de Guadalajara.

(2006). "Vida Académica en las universidades mexicanas: los efectos del trabajo múltiple”. En: Chavoya, María Luisa (coord.). El trabajo académico en la encrucijada de las políticas. Guadalajara: Universidad de Guadalajara, pp.129-144.

Berger, Peter y Thomas Luckman (1994). La construcción social de la realidad. Buenos Aires: Amorrortu.

Bauman, Zygmunt (1990). Pensando sociologicamente. Buenos Aires: Nueva Visión.

Ricoeur (1996). Tiempo y Narración III. México: Siglo XXI.

Weber, Max (1944). Economía y Sociedad. México: Fondo de Cultura Económica. 